\title{
Impacts of Data Uniformity in the Reuse of Acceptance Test Glue Code
}

\author{
Douglas Hiura Longo, Patrícia Vilain and Lucas Pereira da Silva \\ Informatics and Statistics Department, \\ Federal University of Santa Catarina, \\ Florianopolis, Brazil \\ douglas.hiura@posgrad.ufsc.br, patricia.vilain@ufsc.br, lucas.pereira@ufsc.br
}

\begin{abstract}
This paper presents the design and results of an experiment to evaluate the impact/effect of data uniformity in automation of acceptance tests. An experiment to specify acceptance tests, represented by the User Scenarios through User Interaction Diagrams (US-UIDs) format, with non-technical user has been set up involving two projects. In the first project, called $P 1$, the treatment of data uniformity is held by an expert, while in the second project, called $P 2$, no treatment of data uniformity is done. In both projects, automation of acceptance tests was developed for evaluation and comparison of the following artifacts: data uniformity, fixture name sharing, automation time, and glue code volume. The results show that there is a meaningful statistics difference of uniformity between projects P1 and P2, where $P 1$ resulted in a better uniformity. However, although the treatment of data uniformity does not show meaningful statistics difference according to the strategy of fixture names sharing used, the time spent in fixture naming was more than two times higher in P2. In addition, the glue code volume was less than half in P1 comparing to $\mathbf{P 2}$.
\end{abstract}

Keywords-component: Acceptance Test; Agile Software Development; Glue Code; Uniformity; Reuse; US-UID.

\section{INTRODUCTION}

In the agile software development, acceptance tests are adopted to enable the communication and collaboration among the stakeholders [1, 2, 3]. Some agile developers, users and clients use acceptance testing as a way of specifying software requirements instead of using only common artifacts based on natural language $[17,18]$. This is an attempt to improve the quality of requirements because several problems can arise when requirements are written in natural language, for example, readers and writers can use the same word for different concepts, or express the same concept in completely different ways [18]. In addition, it is estimated that $85 \%$ of software defects are originated from ambiguous, incomplete or illusory software requirements [19].

Acceptance tests can be specified using semi-structured formats like user stories (such as Behavior Driven Development - BDD) $[4,5,6]$, tables (such as Fit tables [3, 7]), or diagrams (such as US-UIDs $[8,9,10]$ ). However, the development process of automated acceptance testing is more complex than just specifying acceptance tests in a chosen format. Independent of the acceptance test format, it is also necessary to implement the code that binds each acceptance test to the System Under Test (SUT). This code is known as glue code [16].

In general, acceptance tests are designed to exercise different functions of the SUT. However, in order to put the SUT in a suitable state for execution, the glue code of different acceptance tests may call some common SUT functions. In this way, the reuse of the glue code is important to reduce the time and cost of the maintenance. In line with Borg and Kropp [16], the maintenance of acceptance tests is an issue that appears from the very start of a project. The tests need to be constantly maintained and the test suite should be integrated in a continuous build process [1].

In the agile development it is desirable that users participate in the specification of the acceptance tests, while the testers develop the test automation. It is assumed that a good specification of acceptance tests facilitates the communication among the stakeholders, helping the test automation by the testers. Moreover, specification that involves multiple users can increase the complexity, mainly when users adopt particular data for the tests. For example, the data of an end-of-session requirement can be called "Log out" by one user and "Sign out" by another one. Both data of end-of-session might communicate the intent to the tester, however the ambiguity can lead the tester to a misunderstanding in a way that it is implemented distinct glue code for the same requirement. In this case it is important to uniform the test data before automating the test in order to avoid unnecessary glue code implementation. In [11], Longo and Vilain proposed a metric to measure the data uniformity of acceptance tests in order to detect exaggerated variances in data that may weaken the quality of acceptance tests.

To investigate the impact of data uniformity in automated acceptance tests, in this work we performed an experiment using the User Scenarios through User Interaction Diagrams (USUIDs) format for the acceptance tests. To execute US-UIDs as acceptance tests, it is necessary to bind them to the SUT. The binding between the US-UIDs and the SUT is done through the fixture names and the glue code [10]. Fixture names are labels created a-posteriori of the US-UIDs specifications to bind the US-UID elements to the glue code. The glue code is responsible for exchanging information between the US-UIDs and the SUT during the test execution. While the specification of the USUIDs representing the acceptance testes is a responsibility of the clients and users, the fixture naming and glue code creation is a responsibility of the testers [10]. 
This paper is organized as follow. Section 2 shows details of the problem of data uniformity in the automation of acceptance tests represented by US-UIDs. Section 3 describes the experiment performed and the evaluation methodology. Section 4 presents the results. Finally, Section 5 shows the conclusions.

\section{EXAMPLE OF THE PROBLEM}

The User Scenarios through User Interaction Diagrams (USUIDs) are used for software requirements specifications [8, 9, 10]. These diagrams can be used as automated acceptance tests. The US-UIDs have been suggested as a specialization of the technique UID [20], where the information is replaced with concrete values from the user scenarios. The applicability of the US-UIDs is usually made by non-technical users to create acceptance testing before the development.

In the following we talk about the problem of data uniformity in the acceptance tests represented by US-UIDs. To illustrate this problem, Figure 1 shows a pair of US-UIDs. The pair of USUIDs shown in this example are for specifying requirements of an application similar to WhatsApp. The US-UID A refers to the requirement to respond to a message, and US-UID B refers to the requirement to check status. The example considers two USUIDs with the objective of showing data uniformity problems only in first interaction state (represented by ellipses [8]). The uniformity is calculated comparing data from user inputs (represented by rectangle with string inside [8]) and system outputs (loose strings [8]) between the US-UIDs.

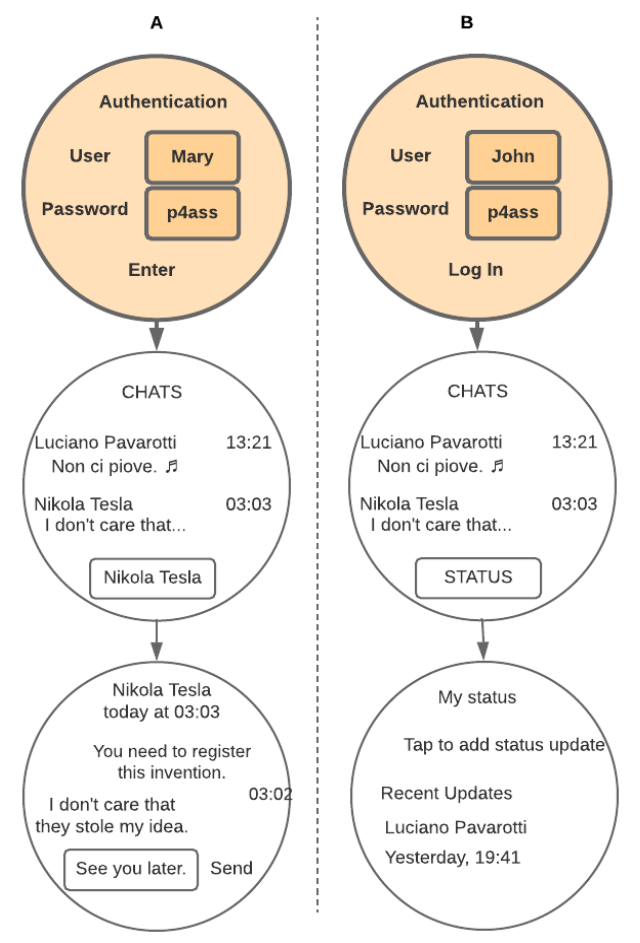

Figure 1. Two US-UIDs with problems of uniformity in data.

The example highlights two problems of data uniformity, where the first one is related to the values of the user inputs "Mary" and "John". These two values are different from each other, but, still, have the same meaning. The meaning is clearly a username. Specially, in US-UIDs with features little known among the stakeholders, this type of non-uniform data might cause loss of meaning leading to miss understandings.

The second problem of data uniformity is related to the system outputs "Enter" (US-UID A, Figure 1) and "Log In" (USUID B, Figure 1). Both system outputs represent, in the SUT implementation, the text of the button for the action for system access. For communication purposes, the stakeholders are able to understand that the text in these two system outputs which, despite the difference, have the same meaning. However, using these US-UIDs as automated tests leads to an unnecessary implementation of the glue code, because the glue code must provide two distinct values ("Enter" and "Log in") for the same requirement.

For the example of the US-UIDs from Figure 1 (considering only the first state of interaction of each US-UID and nonuniform data), three strategies for fixture naming can be developed. These strategies impact in different glue code implementations and also spreads the problems of data uniformity in different glue code structures. The Figure 2 shows three different strategies to naming fixtures and Figure 3 shows de glue code for each strategy.

\section{A. Strategy of Shared Fixture Names (S1)}

The strategy of shared fixture names causes two interaction states (Figure 2, S1) and two user inputs or two system outputs from different US-UIDs share the same fixture name. Figure 2 shows three possible shared fixture names: "AuthenticationState" for the two interaction states, "user" for two user inputs, and "enterButton" for two system outputs. The two interaction states of the US-UIDs from the example are named by "AuthenticationState", sharing, thus, the same fixture name.

The fixture name "user" is used to name the user inputs "Mary" and "John" which represent the user names. Although this fixture name is not the same as the input names, there is no sense of conflict with these names for the example. However, the fixture name "enterButton" for the system outputs "Enter" and "Log In" might create doubts. The proper decision of the developer is to return the US-UIDs to the users or clients and solve which system output name is the most appropriate. However, if the developer keeps the names irregular, the glue code will be developed as shown in Figure 3 (S1).

During the creation of the glue code, it is necessary to implement the class for the interaction states and the methods for each user input and system output in order to bind the US-UIDs to the SUT. The interaction states are linked to the class "AuthenticationState". The method getEnterButton is responsible to bind the SUT to the fixture "enterButton" and to return the result to fulfill the test exercise. In line 6 (S1, Figure 3 ) the SUT is represented only by the string "Enter OR Log In?". In this case a conflict is generated (Figure 3, S1, line 6) because the method may simply return one value, but in US-UIDs there are as candidates the values "Enter" or "Log In", in which, this way, the irregular data is detected due to the conflict on the decision of method return. Therefore, the developer will have a lag upon reviewing the US-UIDs. The reviewing process can take longer and increase the costs if help from users or clients is needed. 


\section{B. Strategy of Fixture Names with Hybrid Sharing (S2)}

The strategy of fixture names with hybrid sharing occurs when two or more interaction states share the same fixture name, and two user inputs or system outputs have different fixture names. Figure 2 (S1) shows the possible fixture names that show a problem of uniformity and both interaction states from USUIDs A and B share the same glue code class. The user inputs "Mary" and "John" have problems of data uniformity but, in practice, they are easily detected. Therefore, in the example of strategy of fixture names with hybrid sharing, the user inputs are not considered. As an example, both interaction states share the fixture "AuthenticationState" and the fixture names "enterButton" and "loginButton" are fixture names that are not shared for the system outputs "Enter" and "Log In". Therefore, the system outputs with similar meaning are handled in different ways. Figure 3 (S2) shows the glue code for the fixture name of hybrid sharing for the example from Figure 2 (S2).
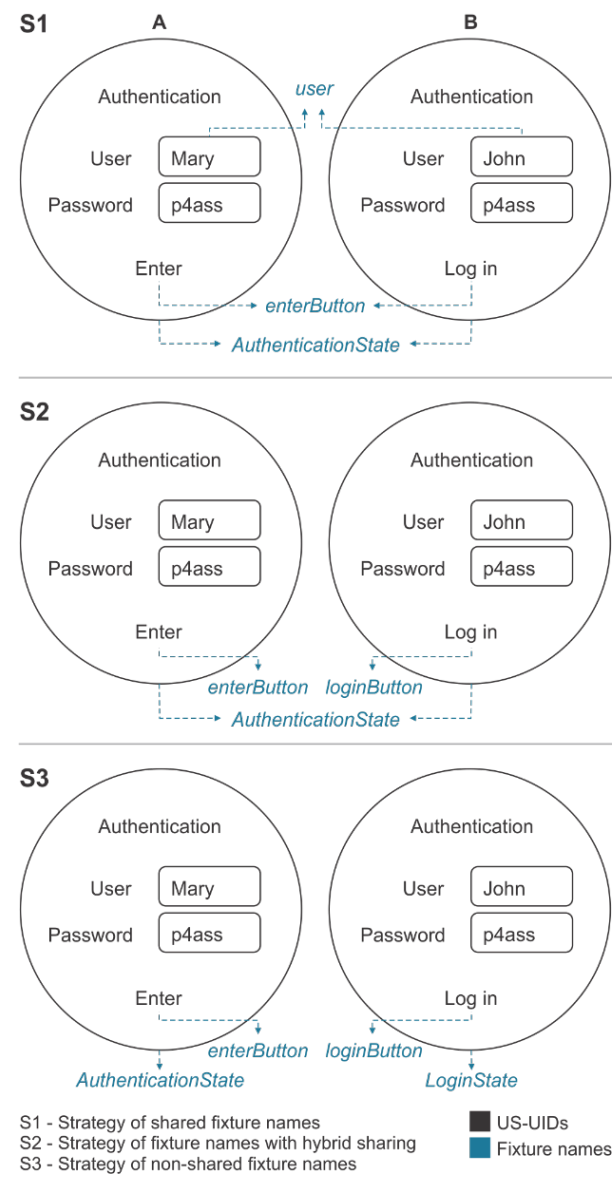

Figure 2. Example of three strategies for fixture naming can be developed.

The fixture code allows the use of a class with non-shared methods, therefore, distinct methods allow returns of different values. The method getEnterButton is responsible for binding the SUT to the fixture "enterButton" and for returning the result to fulfill the test exercise. The method getLoginButton is responsible for binding the SUT to the fixture "loginButton" and for returning the value that fulfills the test exercise. Therefore, both methods have different responsibilities and do not cause any conflicts. It is important to point out that, in this example, both methods return the values from test results just because they are not linked to the SUT yet.

\section{Strategy of Non-Shared Fixture Names (S3)}

The strategy of non-shared fixture names occurs when two interaction states to have different fixture names, as well as two user inputs or two system outputs to have different fixtures. Figure 2 (S3) demonstrates the possible fixture names that show a problem of uniformity.

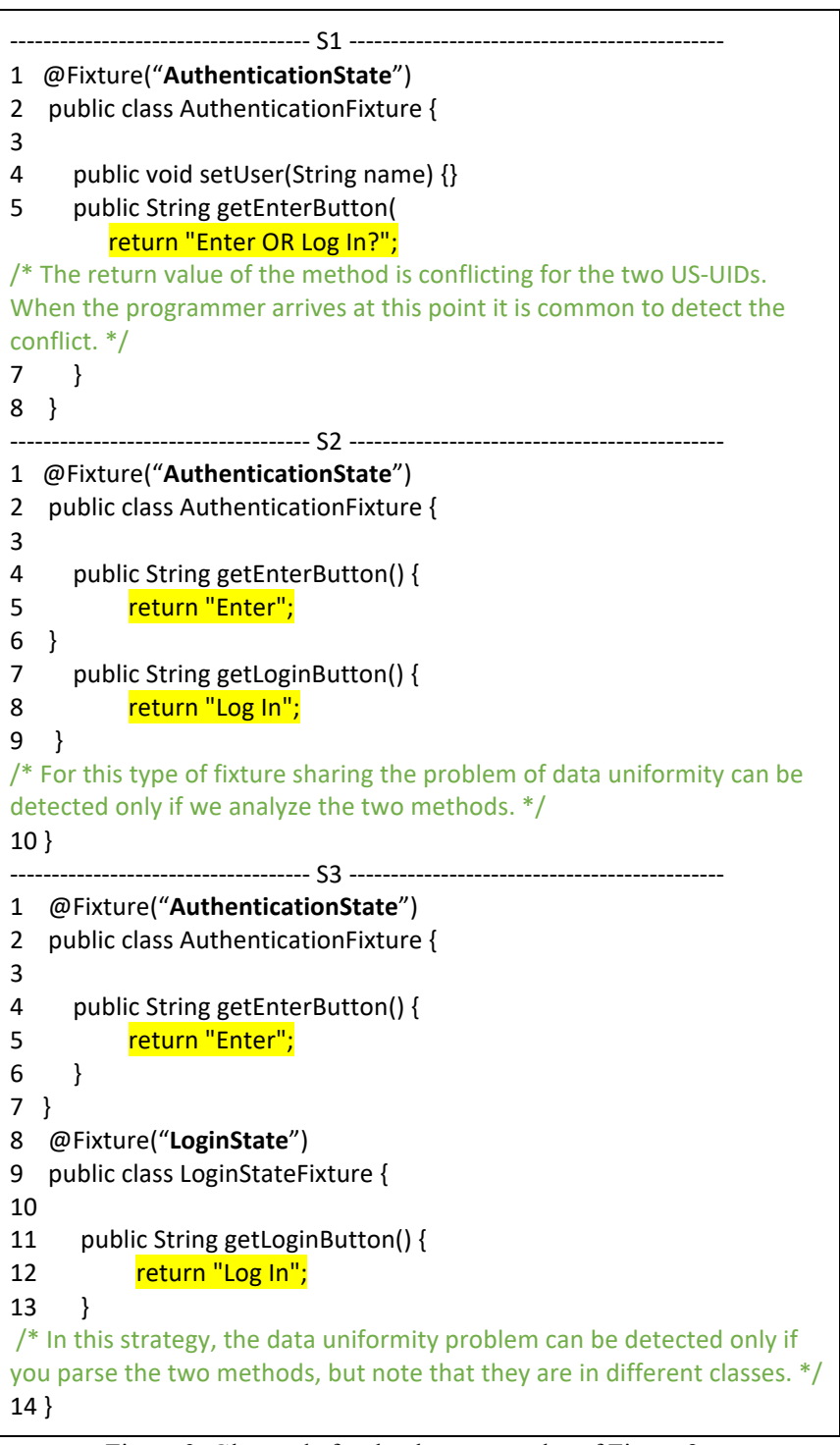

Figure 3. Glue code for the three examples of Figure 2.

In this example we are only considering both interaction states and system outputs "Enter" and "Log In", so there is no fixture name sharing. This way, the interaction states and system outputs with similar meaning are treated in different ways. Figure 3 (S3) shows the glue code for the non-shared fixture names for the example from Figure 2 (S3).

In the level of fixture code, both US-UIDs use two different classes for binding the US-UIDs to the SUT, thus classes and methods are not shared. The class AuthenticationFixture (Figure 2, S3, line 2) contains the methods for the interaction state of US-UID A (Figure 2, S3, left). For the interaction state of USUID B (Figure 2, S3, right) the class LoginState (Figure 2, S3, line 10) is used. This way, the methods for binding the system 
outputs "Enter" and "Log In" to the SUT are allocated in different classes. The method getEnterButton is responsible for binding the SUT to the fixture "enterButton" and for returning the result to fulfill the test exercise. The method getLoginButton is responsible for binding the SUT to the fixture "loginButton" and for returning the result that fulfills the test exercise. Therefore, both methods have different responsibilities and do not cause any conflict. Again, in this example, both methods return the values from test results because they are not linked to the SUT yet.

\section{EXPERIMENT}

The experiment evaluates the treatment of data uniformity in the automation process of acceptance tests using the US-UIDs format. In the experiment, activities such as US-UIDs specification, fixtures naming, and glue code creation are elaborated. The purpose is to investigate the following research questions:

- RQ1: Does the help of an expert in the US-UIDs specification improve the data uniformity?

- $\quad$ RQ2: Does the treatment of uniformity affect any of the strategies of fixture name sharing?

- RQ3: How long does the fixture naming take? How much of the volume of glue code is implemented?

\section{A. Methodology for Evaluation}

For the evaluation, a specification of US-UIDs from requirements of a messaging system has been considered. Figure 4 shows an activities diagram with the three steps of the evaluation: preparation, experiment and result analysis.

1) Preparation. In the preparation step, the participants are trained on the language of the US-UIDs for about 15 minutes; this training is the same as the adopted by Longo and Vilain [8, 9]. During the preparation, non-technical participants are oriented to use pencil, rubber and paper for a small training. After the training with the US-UID, explanations on the messaging system are given. The participants are requested to figure out requisites for an application similar to WhatsApp, Telegram, Hangout or Messenger and, then, specify the requisites as US-UIDs.

2) Experiment: In order to answer the questions and to analyze the treatment of data uniformity in the specification of US-UIDs by non-technical ${ }^{l}$ participants, the specifications are divided in two projects ( $P 1$ and $P 2$ ). In the project $P 1$, the specification is done by one participant. After the specifying, an expert ${ }^{2}$ checks the data uniformity for each US-UID. If the USUID does not show data uniformity, it is then returned to the participant so that he may improve the uniformity according to the expert guidelines. If, in the evaluation from an expert, the US-UID shows a good data uniformity, then the US-UID specification is finished. In the project $P 2$, the specification is done by four non-technical participants without the expert's help. After the specification step is finished, the activity of fixture naming is stated, which is carried out by only one tester. The time of this activity is timed, as it also shows the tester effort to understand the US-UIDs. For all the US-UIDs, the fixtures are named, and this information is necessary for binding the US-UIDS elements to the glue code. After the activity of fixture naming is finished, the activity of glue code creation is carried out. An algorithm has been implemented in order to generate the glue code. The algorithm has the US-UIDs as an input and generates the glue code according to the framework proposed by Longo et al. [10].

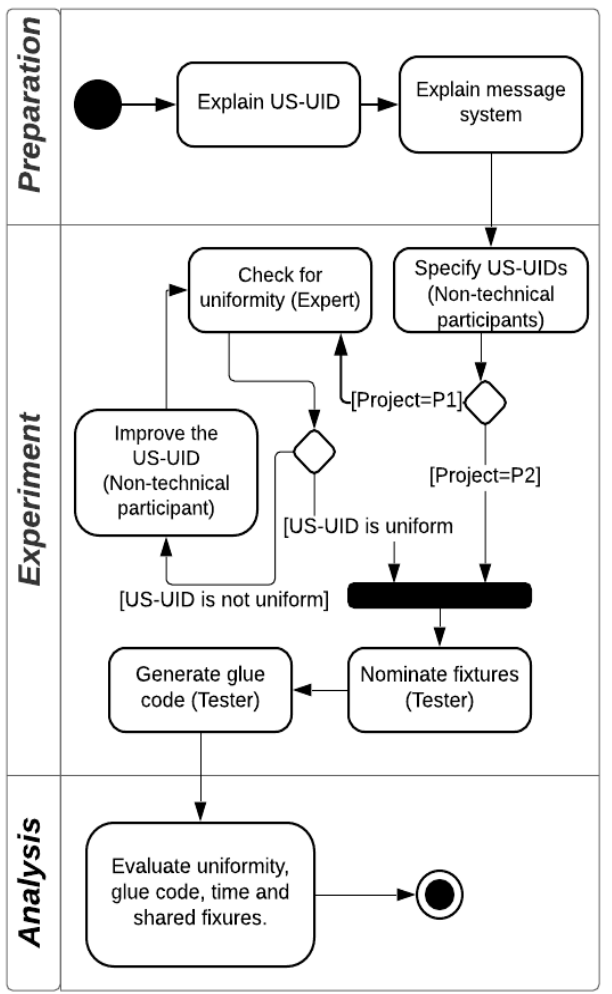

Figure 4 . Activity diagram for uniformity evaluation.

\section{RESULTS}

\section{A. Descriptive Measures of the US-UIDs}

In calculating the measures, all the US-UIDs specified by the participants have been added. This way, there are differences in the descriptive measures of US-UIDs that specify the same requirement, as the specification way of each participant is different. In the project P1, 6 US-UIDs, 31 interaction states, 126 system outputs, 43 user inputs and 25 transitions have been specified. Whereas in project the P2, 20 US-UIDs, 59 interaction states, 308 system outputs, 66 user inputs and 39 interaction states have been specified. There are no doubts that differences exist in the number of elements of the US-UIDs, but it highlights that the intention is specifying the same requirement in both projects.
${ }^{1}$ Non-technical participants are individuals without technical training in information technology.
${ }^{2}$ Expert is an experienced developer with US-UIDs. 


\section{B. Data Uniformity of the US-UIDs (RQ1)}

In order to evaluate the data uniformity between both projects, a metric of data uniformity proposed by Longo and Vilain [11], is used. The metric compares pairs of US-UIDs and the results in a quantitative value. The value measured is in the interval $[0 \%, 100 \%]$, where $0 \%$ indicates total irregularity and $100 \%$ indicates total uniformity. In order to apply the metrics, the US-UIDs resulted from experiment, and that were described on paper, are scanned to the tool $\mathrm{Sc} 3 \mathrm{n} 4 \mathrm{r} 10^{3}$ and, then, the metric is applied computationally. Question RQ1 can be answered by the following formula with the following hypothesis:

$$
\begin{aligned}
& \text { H0: Uniformity } y_{p 1}=\text { Uniformity }_{p 2} \\
& \text { H1: Uniformity } y_{p 1} \neq \text { Uniformity }_{p 2}
\end{aligned}
$$

Uniformity $_{p 1}$ is the uniformity measure of the US-UIDs from project P1; Uniformity $p_{2}$ is the uniformity measure of the US-UIDs from project $\mathrm{P} 2$. The decision for accepting $\mathrm{H} 0$ or $\mathrm{H} 1$ is made according to the data collected from the experiment. The decision for accepting the hypothesis $\mathrm{H} 0$ means that the adding of the treatment of uniformity by the expert has no effect in the specification. However, when it accepts the hypothesis H1, it states that the uniformity is different between both projects, thus it is necessary to compare the uniformity averages to discover which one is the best.

The distribution of the data uniformity was analyzed through a Z-Test, at a level of significance of $(\alpha=0.05)$, obtaining a meaningful statistic difference among the uniformity of both projects $\left(\mathrm{P}_{\text {value }}=2 \cdot 2 \mathrm{e}^{-16}\right)$. Therefore, then with the inclusion of the expert, there is significant difference in the uniformity of the USUIDs data in both projects (H1).

Figure 5 shows the data uniformity in both projects. Project P1 generated 30 pairs of US-UIDs with uniformity average of $64 \%$, median of $67 \%$ of uniformity, and uniformity interval between $[32 \%, 100 \%]$. Project P2 generated 380 pairs of USUIDs with uniformity average of $12 \%$, median of $6 \%$ of uniformity, and uniformity interval between $[0 \%, 42 \%]$, with some discrepant values above this interval. The discrepant values occurred by US-UIDs where the participant had noticed the importance of keeping data uniformity in order to better specify the US-UIDs.

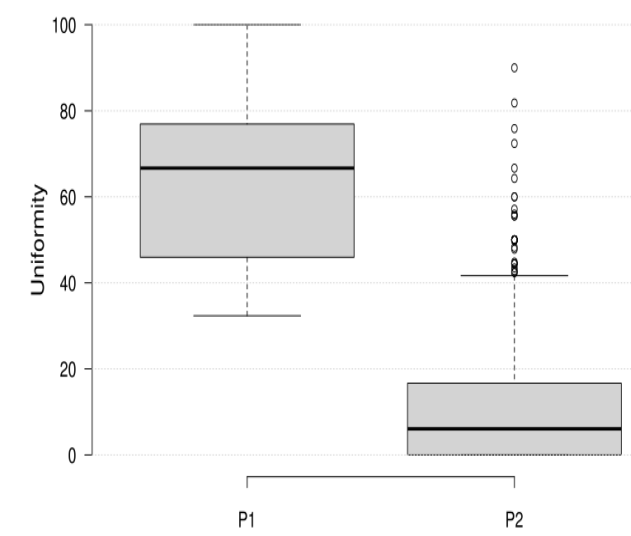

Figure 5. Uniformity of each project per pair of US-UIDs.

\section{Fixture Name Sharing (RQ2)}

The strategies of fixture name sharing were measured by the times that each fixture name is shared, sectioned by interaction states, system outputs and user inputs. For example, according to Figure 2 (S1), the fixture name "user" is shared two times, i.e., "user" is shared by the user inputs "Mary" and "John." For the system outputs and interaction states the count is the same. The count was applied to each project with the aid of an algorithm implemented together with tool Sc3n4r10. Question RQ2 can be answered by a hypothesis test about the number of elements that share the same fixture name with the following hypothesis:

$$
\begin{aligned}
& \text { H0: FixtureNameSharing } p_{p 1}=\text { FixtureNameSharing }_{p 2} \\
& \text { H1: FixtureNameSharing } \\
& p_{1} \neq \text { FixtureNameSharing }
\end{aligned}
$$

FixtureNameSharing ${ }_{p 1}$ is the number of times each fixture name is shared in US-UIDs from project P1; FixtureNameSharing $_{p 2}$ is the number of times each fixture name is shared from project $\mathrm{P} 2$.

The hypothesis can be formulated for fixture name shares of user inputs, system outputs, and interaction states. The decision for accepting $\mathrm{H} 0$ means that the help of an expert has no effect in the strategies of fixture sharing adopted by the tester. However, by accepting the hypothesis $\mathrm{H} 1$, it is affirmed that the strategies of fixture sharing adopted by the tester are different between both projects. Table 1 shows the statistics analysis about the fixture name sharing for each element type. For each element type, the statistic tests shown suggest that there is no meaningful difference in the fixture sharing between projects P1 and $\mathrm{P} 2$. Therefore, the treatment of uniformity does not affect the strategies of fixture sharing for all element type (H0).

TABLE 1. STATISTICAL ANALYSIS.

\begin{tabular}{llcl}
\hline \multicolumn{1}{c}{ Elements } & Test & P value & Statistical Decision $(\boldsymbol{\alpha}=\mathbf{0 . 0 5})$ \\
\hline Interactions & U Test & 0.1301 & $\mathrm{H} 0$ \\
Outputs & Z Test & 0.4426 & $\mathrm{H} 0$ \\
Inputs & U Test & 0.0685 & $\mathrm{H} 0$ \\
\hline
\end{tabular}

\section{Effort (RQ3)}

The effort for fixture naming by the tester is analyzed through time data. Therefore, it is necessary to compare the time spent in each project. The glue code volume also indicates indirectly the effort of the activity of fixture naming.

Figure 6 shows the glue code volume and the time spent in the activity of fixture naming by the tester. Project P1 resulted in 255 lines of code and took 240 minutes to name the fixtures. Project P2 resulted in 834 lines of code and took 552 minutes to name the fixture.

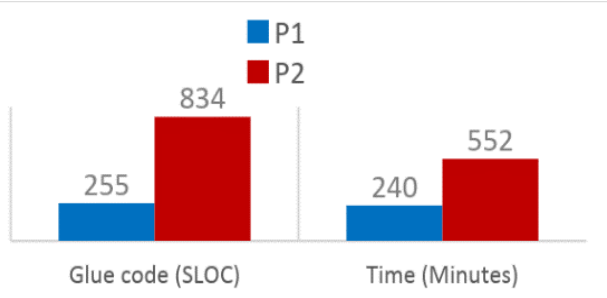

Figure 6. Measures of code volume and time.

\footnotetext{
${ }^{3}$ https://github.com/douglashiura/us-uid.git
} 


\section{CONCLUSIONS}

This paper proposes the treatment of data uniformity in the activity of specifications of the US-UIDs and evaluates the effect of this data uniformity in the glue code reuse. In the evaluation, the treatment of uniformity was done by a participant who is an expert during the activities where non-technical participants specify the US-UIDs. In fact, the result of the treatment by the expert increase the uniformity, i.e., there is a meaningful statistic difference between the project with the treatment of uniformity done by the expert and a project without the same. However, in the activity of fixture naming, developed by the tester, the difference of data uniformity does not affect the fixture name sharing, i.e., the uniformity is not a factor that inclines the tester in the choosing of strategies of fixture name sharing. The developing time of the activity by the tester is very different between a project with uniformity treatment and a project without treatment. Although the treatment of uniformity does not show any influence in the strategies of fixture name sharing, the tester spends more time understanding and naming the fixture in a proper way; the time difference is twice more for a project that does not treat the data uniformity. The glue code volume is also very different. A higher code volume indicates less reuse and more effort in the activity of maintenance. Thus, it is concluded that data uniformity should be addressed at the time of US-UIDs specification, as these later saves time for the naming of fixtures and significantly reduces the amount of glue code.

As a threats of validity it is important to emphasize that this work considered only the initial requirements of a messaging system, so the results may be different for a scope of applications with lots of requirements. However, it is important to maintain uniformity in project implementation because the tests are more comprehensive, which impacts in less time to develop them (although there is interaction between developers and customers for uniformization) and less glue code to maintain during development. Yet, the software development challenges from the real world, many times, rely on projects with more stakeholders, that way we need investigate the problem in projects with different amounts of participants.

Other works address the quality improvement on specifying user stories. Lucassen et al. [13, 14] proposed a framework to evaluate fourteen quality criteria. These criteria are adopted in the Grimm Method [15] and applied in a case of study in the industry. However, the applying of the Grimm Method did not result in a meaningful difference in the software development process.

Finally, the reliability of an experiment refers to the capacity of other researchers to reply the methodology [12]. For the replication, the methodology has been detailed and algorithms for uniformity measuring and fixture sharing along with the tool Sc3n4r10 have been made available. This study was financed in part by the Coordenação de Aperfeiçoamento de Pessoal de Nível Superior - Brasil (CAPES) - Finance code 001.

\section{REFERENCES}

[1] B. Haugset and G. K. Hanssen, Automated acceptance testing: A literature review and an industrial case study. Agile, 2008. AGILE'08. Conference, IEEE, pp. 27-38, 2008
[2] T. Dybå and T. Dingsøyr, Empirical studies of agile software development: A systematic review. Information and software technology. Elsevier, vol. 50, pp. 833-859, 2008.

[3] G. K. Hanssen and B. Haugset, "Automated acceptance testing using fit" in System Sciences, HICSS'09, 42nd Hawaii International Conference on. IEEE, pp. 1-8, 2009.

[4] Cohn, Mike. User stories applied: For agile software development. Addison-Wesley Professional, 2004.

[5] Wautelet, Y., Heng, S., Kolp, M., \& Mirbel, I. "Unifying and extending user story models" in International Conference on Advanced Information Systems Engineering. Springer, Cham, pp. 211-225, 2014.

[6] Lucassen, G., Dalpiaz, F., van der Werf, J. M. E., \& Brinkkemper, S. "The use and effectiveness of user stories in practice". in International Working Conference on Requirements Engineering: Foundation for Software Quality. Springer, Cham. p. 205-222, 2016.

[7] Druk, Michael, and Martin Kropp. "ReFit: A Fit test maintenance plug-in for the Eclipse refactoring plug-in." in Developing Tools as Plug-ins (TOPI), 2013 3rd International Workshop on. IEEE, pp. 7-12, 2013.

[8] D. H Longo., and P. Vilain. User scenarios through user interaction diagrams. International Journal of Software Engineering and Knowledge Engineering 25.09n10, pp. 1771-1775, 2015.

[9] D. H Longo., and P. Vilain. Creating User Scenarios through User Interaction Diagrams by Non-Technical Customers. in Software Engineering and Knowledge Engineering - SEKE15. KSI Research Inc. and Knowledge Systems Institute Graduate School, pp.330-335, 2015.

[10] Longo, D. H., Vilain, P., da Silva, L. P., and Mello, R. D. S. A web framework for test automation: user scenarios through user interaction diagrams. in Proceedings of the 18th International Conference on Information Integration and Web-based Applications and Services. ACM, pp 458-467, 2016.

[11] D. H Longo., and P. Vilain. Metrics for Data Uniformity of User Scenarios through User Interaction Diagrams". Software Engineering and Knowledge Engineering - SEKE18. KSI Research Inc. and Knowledge Systems Institute Graduate School, pp.1-6, 2018.

[12] A. K. Massey, R. L. Rutledge, A. I. Anton, P. P. Swire. Identifying and classifying ambiguity for regulatory requirements, " Requirements Engineering Conference (RE), 2014 IEEE 22nd International, IEEE, pp. $83-92,2014$.

[13] Lucassen, G., Dalpiaz, F., van der Werf, J. M. E., and Brinkkemper, S. Improving agile requirements: the quality user story framework and tool. Requirements Engineering, v. 21, n. 3, p. 383-403, 2016.

[14] Lucassen, G., Dalpiaz, F., van der Werf, J. M. E., and Brinkkemper, S. Forging high-quality user stories: towards a discipline for agile requirements. in Requirements Engineering Conference (RE), 2015 IEEE 23rd International. IEEE. p. 126-135, 2015.

[15] Lucassen, G., Dalpiaz, F., van der Werf, J. M. E., and Brinkkemper, S. Improving user story practice with the Grimm Method: A multiple case study in the software industry. in International Working Conference on Requirements Engineering: Foundation for Software Quality. Springer, Cham, 2017.

[16] Borg, Rodrick; Kropp, Martin, "Automated acceptance test refactoring". in Proceedings of the 4th Workshop on Refactoring Tools. ACM. p. 1521, 2011.

[17] Dos Santos, E. C.; P. Vilain, “Automated Acceptance Tests as Software Requirements: An Experiment to Compare the Applicability of Fit Tables and Gherkin Language". In. International Conference on Agile Software Development. Springer, Cham. p. 104-119, 2018.

[18] Sommerville, I.: Software Engineering. 9th edn. Pearson Education, Boston 2015.

[19] Torchiano, M., Ricca, F., M. D. Penta, "Talking tests": a preliminary experimental study on fit user acceptance tests. in First International Symposium on Empirical Software Engineering and Measurement (ESEM 2007), p. 464-466, 2007.

[20] Zeferino, N. V., and Vilain, P. "A model-driven approach for generating interfaces from user interaction diagrams" in Proceedings of the 16th International Conference on Information Integration and Web-based Applications \& Services. ACM, 474-478, 2014. 